\title{
The Effect of Paricalcitol on Vascular Calcification and Cardiovascular Disease in Uremia: Beyond PTH Control
}

\author{
Mario Cozzolino, Florjan Mehmeti, Paola Ciceri, Elisa Volpi, Andrea Stucchi, \\ Irene Brenna, and Daniele Cusi \\ Renal Division, DMCO, San Paolo Hospital, School of Medicine, University of Milan, Via A. di Rudinì 8; 20142, Milan, Italy \\ Correspondence should be addressed to Mario Cozzolino, mariocozzolino@hotmail.com
}

Received 30 October 2010; Accepted 18 January 2011

Academic Editor: Biagio Raffaele Di Iorio

Copyright ( $) 2011$ Mario Cozzolino et al. This is an open access article distributed under the Creative Commons Attribution License, which permits unrestricted use, distribution, and reproduction in any medium, provided the original work is properly cited.

Secondary hyperparathyroidism is a systemic disorder that associates with bone and cardiovascular disease, including arterial calcification. Treatment with calcitriol, the active form of vitamin D, reduces parathyroid hormone levels, but may result in elevations in serum calcium and phosphorus, increasing the risk of vascular calcification in dialysis patients. New vitamin D receptor activators (VDRAs) have been developed and investigated with the rationale to treat high serum PTH levels, with a reduced risk of hypercalcemia and hyperphosphatemia. Paricalcitol is a selective VDRA that suppresses PTH secretion with minimal increases on serum calcium and phosphate. Moreover, paricalcitol prevents vascular calcification in experimental models of renal failure, compared with calcitriol.

\section{Introduction}

Secondary hyperparathyroidism (SHPT) affects less than half of the approximately 320000 haemodialysis (HD) patients in the US who suffer from stage- $V$ chronic kidney disease (CKD) (also known as end-stage renal disease or end-stage kidney disease) [41]. SHPT is characterized by abnormally elevated serum concentrations of intact parathyroid hormone (PTH), and abnormalities of serum calcium, phosphorous, and vitamin $\mathrm{D}$ concentrations. Inadequate treatment of SHPT has been associated with the following conditions: skeletal abnormalities (renal osteodystrophy), cardiovascular complications [1], infections and immunoregulatory dysfunction [2], foot and extremity complications, and anaemia. Patients on HD are at increased risk for fracture (including hip fracture) and vascular calcification, which results in significant morbidity (including hospitalization) and mortality [3].

Diminished levels of vitamin D have been significantly associated with an increased risk of vascular calcification in patients with a moderate or high risk of coronary heart disease, even with normal renal function [4]. Age, race, diabetic history, and $\log 1,25-\mathrm{D}$ have been related to arterial mass of coronary calcium and inversely correlated with calcium phosphate mass. Furthermore, a cross-sectional examination from NHANES (2001-2004) patient data demonstrated a significant graded, inverse correlation between serum 25D levels and the prevalence of peripheral arterial disease and was independent of gender, age, race, and multivariable adjustment [5].

Several studies in patients with CKD have now correlated arterial calcification with the presence of coronary artery disease [6], peripheral vascular disease [7], left ventricular hypertrophy [6] and mortality. Increased pulse pressure, left ventricular hypertrophy, and arrhythmias, resulting from arterial stiffness, have been suggested as potential effects of arterial calcification that may lead to cardiovascular disease and death.

CKD patients have a dramatically higher incidence of cardiovascular morbidity and mortality compared to the general population. Studies show that CVD mortality is 10 to 70 times greater in the dialysis population than in the general age-matched cohort [8].

In the last 10 years, several studies pointed out that vascular calcification is a major cause of cardiovascular disease in the dialysis population. In CKD patients, high levels of 
plasma calcium, serum phosphate, and PTH play a critical role in the pathogenesis of cardiovascular events [9]. Nasri et al. [10] analyzed the influence of PTH on myocardial function. In their cross-sectional study in hemodialysis patients, they determined that excess PTH played a significant role in the development of $\mathrm{LVH}$ and reduced left ventricular ejection fraction. In a decade-long retrospective study, Dai et al. found a $52 \%$ prevalence of severe LVH among ESRD patients. Ha et al. [11] further extended the links between $\mathrm{LVH}$ and CKD, finding an $87 \%$ prevalence of concentric and eccentric LVH among predialysis patients. Foley et al. corroborated the results in a decade-long prospective study, finding that $80 \%$ of the 433 patients initiating dialysis presented with LVH on echocardiography. The relationship between elevated PTH and LVH was further explored in a retrospective study by Goto et al. [12] who determined that parathyroidectomy in CKD patients with advanced SHPT led to a significant improvement of left ventricular ejection, fraction, and function. Hyperphosphatemia and hypercalcemia have been shown to promote calcification of the vasculature, myocardium, and cardiac valves. Vascular calcification manifested in reduced vessel wall elasticity, increased intimamedia layer thickness, and enhanced pulse-wave velocityhas been linked to $\mathrm{LVH}$ - and occurs with increased severity in dialysis patients versus non-CKD patients.

Patients develop extensive medial calcification, which causes increased arterial stiffness and high morbidity and mortality due to cardiovascular events [13]. A variety of risk factors are associated with vascular calcification in dialysis patients (time on dialysis, uremic toxins, history of diabetes, inflammation), but abnormal ties in bone mineral metabolism may play a critical role [14]. In fact, elevated serum phosphate, calcium-phosphate product, and PTH levels contribute to vascular calcification, although their roles are incompletely understood $[15,16]$.

Elevations of serum phosphate and calcium-phosphate product levels may worsen cardiovascular events in the uremic population, through a progressive increase in calcium deposition in the coronary arteries and heart valves [8]. Furthermore, calcium-containing phosphate binders can increase calcium load [17]. In haemodialysis children and young adults, Goodman et al. [18] showed a correlation between coronary artery calcification detected by electron beam computed tomography (EBCT) and years on dialysis, serum phosphorus, calcium-phosphate product levels, and daily intake of calcium. Moreover, experimental observations in uremic rats $[19,20]$ and in dialysis patients $[21]$ indicate that the calcium and aluminium-free phosphate binder, Sevelamer $\mathrm{HCl}$, reduced the progression of cardiovascular calcification observed in a control group treated with calcium-based phosphate binders [20].

\section{The Role of Paricalcitol on CKD-MBD Prevention and Treatment}

Prevention and treatment of SHPT commonly requires control of both phosphate and $1,25(\mathrm{OH})_{2} \mathrm{D}$ levels in serum. Phosphate levels are usually controlled by reducing dietary phosphate intake, by dialysis, and by using phosphatebinders. $1,25(\mathrm{OH})_{2}$ D effectively suppresses PTH production and improves bone histology in some patients [22].

The therapeutic use of $1,25(\mathrm{OH})_{2} \mathrm{D}$ in CKD mainly aims to raise intestinal absorption of calcium, to protect bone against osteomalacia and to control parathyroid function. However, also the function of other organs expressing VDR, as kidney, pancreas, myocardium, and testis, might be improved by vitamin $\mathrm{D}$ administration. $1,25(\mathrm{OH})_{2} \mathrm{D}$ inhibitory effect on PTH synthesis and parathyroid hyperplasia is well established in CKD. Vitamin D analogues with a lower hypercalcemic and hyperphosphatemic activity, are now available as an efficient alternative to $1,25(\mathrm{OH})_{2} \mathrm{D}$ treatment. Furthermore, normal serum levels of $25(\mathrm{OH}) \mathrm{D}$ are relevant for the prevention or treatment of SHPT, due to the capacity of $25(\mathrm{OH}) \mathrm{D}$ to support renal $1 \alpha$-hydroxylase activity or the synthesis of active vitamin $\mathrm{D}$ metabolites different from $1,25(\mathrm{OH})_{2} \mathrm{D}$.

Clinical targets for serum concentrations of phosphate, calcium, and PTH have been proposed in the 2003 guidelines proposed by the Kidney Dialysis Outcomes Quality Initiative (K/DOQI) Work Group [23]. Target for plasma concentrations of PTH increases according to creatinine clearance because patients develop a progressive tissue resistance to PTH explained by a diminished expression of PTH receptors and by $1,25(\mathrm{OH})_{2} \mathrm{D}$ deficiency or resistance $[24,25]$. The low target for calcium concentrations aims to avoid calcium overload and excessively high calcium $\mathrm{x}$ phosphate product.

A particular bone complication of the therapy with vitamin D metabolites occurs in case of excessive parathyroid suppression. As defined in the K/DOQI guidelines, the target for PTH concentration is $150-300 \mathrm{pg} / \mathrm{mL}$ in end-stage CKD. The new K/DIGO guidelines proposed a new target for serum PTH levels [26], but if the values of circulating PTH are suppressed below these limits, bone cells become quiescent and bone remodelling is far slowed [27]. The histology pattern of these functional alterations is adynamic bone disease, characterized by decreased number of bone cells, bone metabolic inactivity, and reduced bone formation rate [25]. The mechanism of adynamic bone disease is unknown. The clinical consequences of adynamic bone disease may be an increased fracture risk while its relationship with osteoporosis is still debated [28]. At the opposite, osteomalacia may develop in case of $1,25(\mathrm{OH})_{2} \mathrm{D}$ deficiency, even though the toxic effect of aluminum overload has been the most frequent cause of osteomalacia in dialysis population till some years ago $[29,30]$.

Several new vitamin D analogues have been developed and investigated with the rationale to treat secondary HPT with a reduced risk of hypercalcemia and hyperphosphatemia in CKD patients. Vitamin D analogues have variable affinity for the components of the vitamin D system, including the vitamin D-binding protein and the nuclear VDR. Some of the effects are genomic and mediated through changes in the structural configuration of the vitamin DVDR complex or in the affinity of the vitamin D-VDR complex for the key response elements in various target genes. There are currently 3 vitamin D analogues approved for use in CKD patients with secondary HPT in the US, 
1,25-dihydroxy-22-oxavitamin $\mathrm{D}_{3}$ (22-oxacalcitriol, OCT), 1,25-dihydroxy-19-norvitamin $\mathrm{D}_{2}$ (19-norD 2 , paricalcitol), and $1 \alpha$-hydroxyvitamin $\mathrm{D}_{2}\left(1 \alpha \mathrm{OHD}_{2}\right)$.

Three identical, 12-week studies determined that paricalcitol was safe and effective in suppressing serum PTH levels in $78 \mathrm{HD}$ patients without significantly increasing serum calcium and phosphorus levels. The mean percentage decrease in iPTH was $60 \%$, and calcium levels rose slightly but remained within the normal range. Changes in phosphate from baseline to study end were not significantly different between the group treated with paricalcitol and the group treated with placebo.

First, Martin et al. [31] reported the outcomes of up to 1 year of therapy with paricalcitol in 164 HD patients. Paricalcitol rapidly suppressed PTH, with the mean iPTH reaching a target of $150-300 \mathrm{pg} / \mathrm{mL}$ by month 5 . Furthermore, 19norD $\mathrm{D}_{2}$ had minimal effects on calcium and phosphate. Most (70\%) of the episodes of hypercalcemia ( $>11.5 \mathrm{mg} / \mathrm{dL})$ that occurred did so when iPTH was suppressed below $150 \mathrm{pg} / \mathrm{mL}$.

Second, a 12-week study by Martin et al. [32] compared PTH suppression and calcium/phosphate outcomes when $125 \mathrm{HD}$ patients were given doses of paricalcitol that were based on the patient's dry weight or on the serum level of iPTH. Both regimens were effective in reducing iPTH to the target of four consecutive weeks of $30 \%$ or greater reductions from baseline. The patients receiving the dosing regimen based on iPTH level reached this target significantly faster than those on the weight-based regimen (31 versus 45 days, $P=.0306)$. No incidences of hypercalcemia occurred in either group.

Third, Llach and yudd [30] proved that $19-$ norD $_{2}$ is effective in suppressing PTH in $37 \mathrm{HD}$ calcitriol-resistant patients. This study showed that using $3 \mathrm{mcg}$ of paricalcitol for each $1 \mathrm{mcg}$ of calcitriol that the patient had previously received (a $1: 3$ ratio) resulted in a decline in mean $\mathrm{iPTH}$ from 836 to $341 \mathrm{pg} / \mathrm{mL}$ after 2 months. Clearly the above studies establish that $19-$ norD $_{2}$ rapidly suppresses $\mathrm{PTH}$ with no significant difference in the incidence of hyperphosphatemia or hypercalcemia compared to placebo.

Paricalcitol has also been directly compared with calcitriol in an international study involving HD 263 patients. This study showed that paricalcitol reduced iPTH $50 \%$ or more from baseline and did so significantly faster than calcitriol. Patients receiving paricalcitol had significantly fewer sustained episodes of hypercalcemia and/or increased $\mathrm{Ca} \times \mathrm{P}$ product [33]. In addition, a historical study examined the records of 67,399 patients who had been treated exclusively with calcitriol or paricalcitol. Patients were evaluated over a 2-year period until one of the following occurred: death, a switch to another vitamin D formulation, renal transplantation, or transfer to another facility. This study showed a statistically significant difference in survival rate in favor of paricalcitol over calcitriol [34]. Furthermore, in patients taking $19-$ norD $_{2}$ a reduced hospitalization rate was observed [35]. These findings will need to be supported by prospective trials before definitive conclusions can be made.

The mechanism for paricalcitol's low calcemic effect is due to reduced stimulation of intestinal calcium transport

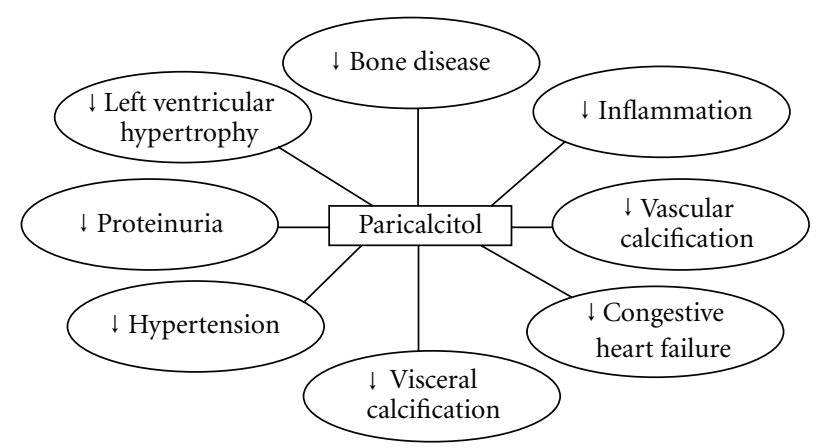

FIGURE 1

proteins (e.g., calbindin, CAT, and PMAT) compared to calcitriol, [36, 37] thus conferring a selective activator function [38].

With the ubiquitous presence of the VDR in many tissues, it is of little wonder that the role of the VDR is multifaceted in providing tissue-specific responses. The impact of selective VDR activators on vascular calcification is multifaceted in that in vivo and clinical outcomes show variable results depending on the clinical circumstance and dose of VDR activator administered [39]. Although VDR activation may mitigate the effects of uremia-induced arterial calcification, emerging data support a potential role for VDR activation in preventing or ameliorating the pathogenesis of atherosclerosis.

These are a number of examples of many selective VDR activator effects on cardiovascular structure and function that, if borne out in humans, have the potential to improve clinical outcomes in CKD-MBD (Figure 1).

Low or clinically relevant doses of VDR activators correlate with decreased deposition of calcium and improved therapeutic outcomes, whereas high doses may actually induce vascular calcification. In 28-week-old mice, therapeutically relevant doses of calcitriol and paricalcitol reduced aortic calcium levels and aortic gene expression of osterix, Msx2, core binding factor (alpha)1 (Cbfa1/Runx-2), and osteocalcin, markers for progression of vascular calcification; while an elevated dose of 1,25-D or paricalcitol actually stimulated vascular calcification [40]. In a study of uremic rats, doxercalciferol produced marked increases in aortic calcification and aortic mRNA and protein expression of Runx2 and osteocalcin, whereas paricalcitol had no effect on these endpoints [41].

VDR activation has been shown to have a number of potential ameliorative effects on both inhibitors and inducers of calcification. The addition of VDR activators suppresses calcification inducers type I collagen, bone sialoprotein, IL$1 \beta$, and tumor necrosis factor (TNF)- $\alpha$, as well as bone morphogenetic protein (BMP)-2 [42]. In addition, calcification inhibitors matrix Gla protein (MGP), osteopontin, and osteocalcin were upregulated by VDR activator treatment [43]. The same is true for type IV collagen.

Another hypothetical mechanism of VDR activation impact on cardiovascular outcome is an inappropriate activation of the renin-angiotensin-aldosterone system (RAAS), 
which plays a central role in the regulation of blood pressure, electrolyte, and volume homeostasis and may represent a major risk factor for hypertension, heart attack, and stroke. Evidence from clinical studies has demonstrated an inverse relationship between circulating vitamin $\mathrm{D}$ levels and the blood pressure and/or plasma renin activity [44].

\section{Conclusions}

The modern strategies to prevent SHPT in CKD patients give great relevance to vitamin D replacement therapy. A sound approach to treatment requires taking into account many factors, including stage of CKD, underlying renal disorder, levels of circulating PTH, bone status, vitamin D deposits, and serum calcium and phosphate levels. The aim of vitamin D replacement therapy is to prevent SHPT from the early stages of CKD, because once parathyroid hyperplasia and osteodystrophy develop, they cannot be completely reverted. Because nephrologists have largely focused their attention on dialysis patients, unfortunately few studies have analyzed the outcome of vitamin D therapy in nonuremic patients.

The therapeutic strategies for SHPT are now changing. The availability of selective VDRAs may warrant inhibition of parathyroid glands with lower effect on calcium and phosphate levels and perhaps reduces mortality of dialysis patients. Paricalcitol is a selective VDRA with minor impact on serum $\mathrm{Ca}$ and $\mathrm{P}$ levels. Paricalcitol is growly used in dialysis patients for its efficacy and safety in the treatment and prevention of SHPT in this population. Recent studies demonstrated that therapy with paricalcitol injection is associated with improved survival, fewer hospitalizations, and shorter hospital stays in HD patients compared with calcitriol, even if prospective clinical trials are ongoing. Additionally, subjects on $\mathrm{HD}$ who did not receive active vitamin $\mathrm{D}$ had higher all-cause and cardiovascular mortality compared with subjects who received any form of active vitamin $\mathrm{D}$.

\section{References}

[1] K. Tsuchihashi, H. Takizawa, T. A. Torii et al., "Hypoparathyroidism potentiates cardiovascular complications through disturbed calcium metabolism: possible risk of vitamin $\mathrm{D}_{3}$ analog administration in dialysis patients with end-stage renal disease," Nephron, vol. 84, no. 1, pp. 13-20, 2000.

[2] D. Rubinger, M. M. Friedlaender, and J. Silver, "Progressive vascular calcification with necrosis of extremities in hemodialysis patients: a possible role of iron overload," American Journal of Kidney Diseases, vol. 7, no. 2, pp. 125-129, 1986.

[3] C. O. Stehman-Breen, D. J. Sherrard, A. M. Alem et al., "Risk factors for hip fracture among patients with end-stage renal disease," Kidney International, vol. 58, no. 5, pp. 2200-2205, 2000.

[4] T. J. Wang, M. J. Pencina, S. L. Booth et al., "Vitamin D deficiency and risk of cardiovascular disease," Circulation, vol. 117, no. 4, pp. 503-511, 2008.

[5] J. Kumar, P. Muntner, F. J. Kaskel, S. M. Hailpern, and M. L. Melamed, "Prevalence and associations of 25-hydroxyvitamin D deficiency in US children: NHANES 2001-2004," Pediatrics, vol. 124, no. 3, pp. e362-e370, 2009.
[6] A. Yildiz, E. Memisoglu, H. Oflaz et al., "Atherosclerosis and vascular calcification are independent predictors of left ventricular hypertrophy in chronic haemodialysis patients," Nephrology Dialysis Transplantation, vol. 20, no. 4, pp. 760767, 2005.

[7] M. S. Joy, P. C. Karagiannis, and F. W. Peyerl, "Outcomes of secondary hyperparathyroidism in chronic kidney disease and the direct costs of treatment," Journal of Managed Care Pharmacy, vol. 13, no. 5, pp. 397-411, 2007.

[8] G. M. London, A. P. Guérin, S. J. Marchais, F. Métivier, B. Pannier, and H. Adda, "Arterial media calcification in endstage renal disease: impact on all-cause and cardiovascular mortality," Nephrology Dialysis Transplantation, vol. 18, no. 9, pp. 1731-1740, 2003.

[9] P. Raggi, A. Boulay, S. Chasan-Taber et al., "Cardiac calcification in adult hemodialysis patients: a link between end-stage renal disease and cardiovascular disease?" Journal of the American College of Cardiology, vol. 39, no. 4, pp. 695-701, 2002.

[10] H. Nasri, A. Baradaran, and A. S. A. Naderi, "Close association between parathyroid hormone and left ventricular function and structure in end-stage renal failure patients under maintenance hemodialysis," Acta Medica Austriaca, vol. 31, no. 3, pp. 67-72, 2004.

[11] S. K. Ha, H. S. Park, S. J. Kim, C. H. Park, D. S. Kim, and H. S. Kim, "Prevalence and patterns of left ventricular hypertrophy in patients with predialysis chronic renal failure," Journal of Korean Medical Science, vol. 13, no. 5, pp. 488-494, 1998.

[12] N. Goto, Y. Tominaga, S. Matsuoka et al., "Cardiovascular complications caused by advanced secondary hyperparathyroidism in chronic dialysis patients; special focus on dilated cardiomyopathy," Clinical and Experimental Nephrology, vol. 9, no. 2, pp. 138-141, 2005.

[13] M. Cozzolino, D. Brancaccio, M. Gallieni, and E. Slatopolsky, "Pathogenesis of vascular calcification in chronic kidney disease," Kidney International, vol. 68, no. 2, pp. 429-436, 2005.

[14] M. G. Jakoby and C. F. Semenkovich, "The role of osteoprogenitors in vascular calcification," Current Opinion in Nephrology and Hypertension, vol. 9, no. 1, pp. 11-15, 2000.

[15] D. Brancaccio and M. Cozzolino, "The mechanism of calcium deposition in soft tissues," Contributions to Nephrology, vol. 149, pp. 279-286, 2005.

[16] G. A. Block and F. K. Port, "Re-evaluation of risks associated with hyperphosphatemia and hyperparathyroidism in dialysis patients: recommendations for a change in management," American Journal of Kidney Diseases, vol. 35, no. 6, pp. 1226-1237, 2000.

[17] M. Cozzolino, A. S. Dusso, H. Liapis et al., "The effects of sevelamer hydrochloride and calcium carbonate on kidney calcification in uremic rats," Journal of the American Society of Nephrology, vol. 13, no. 9, pp. 2299-2308, 2002.

[18] W. G. Goodman, J. Goldin, B. D. Kuizon et al., "Coronaryartery calcification in young adults with end-stage renal disease who are undergoing dialysis," The New England Journal of Medicine, vol. 342, no. 20, pp. 1478-1483, 2000.

[19] M. Cozzolino, M. E. Staniforth, H. Liapis et al., "Sevelamer hydrochloride attenuates kidney and cardiovascular calcifications in long-term experimental uremia," Kidney International, vol. 64, no. 5, pp. 1653-1661, 2003.

[20] G. M. Chertow, S. K. Burke, and P. Raggi, "Sevelamer attenuates the progression of coronary and aortic calcification in hemodialysis patients," Kidney International, vol. 62, no. 1, pp. 245-252, 2002.

[21] I. G. Nikolov, N. Joki, J. Maizel, B. Lacour, T. B. Drüeke, and Z. A. Massy, "Pleiotropic effects of the non-calcium phosphate 
binder sevelamer," Kidney International, vol. 70, no. 105, pp. S16-S23, 2006.

[22] D. Coyne, M. Acharya, P. Qiu et al., "Paricalcitol capsule for the treatment of secondary hyperparathyroidism in stages 3 and 4 CKD," American Journal of Kidney Diseases, vol. 47, no. 2, pp. 263-276, 2006.

[23] S. G. Massry, J. W. Coburn, G. M. Chertow et al., "K/DOQI clinical practice guidelines for bone metabolism and disease in chronic kidney disease," American Journal of Kidney Diseases, vol. 42, no. 3, pp. S63-S200, 2003.

[24] S. Disthabanchong, H. Hassan, C. L. McConkey, K. J. Martin, and E. A. Gonzalez, "Regulation of PTH1 receptor expression by uremic ultrafiltrate in UMR 106-01 osteoblast-like cells," Kidney International, vol. 65, no. 3, pp. 897-903, 2004.

[25] G. Hercz, C. Greenwood, Y. Pei et al., "Aplastic osteodystrophy without aluminum: the role of 'suppressed' parathyroid function," Kidney International, vol. 44, no. 4, pp. 860-866, 1993.

[26] KDIGO, "KDIGO clinical practice guideline for the diagnosis, evaluation, prevention, and treatment of chronic kidney disease-mineral and bone disorder (CKD-MBD)," Kidney International, vol. 76, supplement 113, 2009.

[27] M. Coco and H. Rush, "Increased incidence of hip fractures in dialysis patients with low serum parathyroid hormone," American Journal of Kidney Diseases, vol. 36, no. 6, pp. 1115-1126, 2000.

[28] G. Gournot-Witmer, J. Zingraff, and J. J. Plachot, "Aluminum localization in bone from hemodialyzed patients: relationship to matrix mineralization," Kidney International, vol. 20, no. 3, pp. 375-385, 1981.

[29] D. Hernandez, M. T. Concepcion, V. Lorenzo et al., "Adynamic bone disease with negative aluminium staining in predialysis patients: prevalence and evolution after maintenance dialysis," Nephrology Dialysis Transplantation, vol. 9, no. 5, pp. 517-523, 1994.

[30] F. Llach and M. Yudd, "Paricalcitol in dialysis patients with calcitriol-resistant secondary hyperparathyroidism," American Journal of Kidney Diseases, vol. 38, no. 5, pp. S45-S50, 2001.

[31] K. J. Martin, E. A. González, M. Gellens, L. L. Hamm, H. Abboud, and J. Lindberg, "19-nor-1- $\alpha$-25-dihydroxyvitamin D (paricalcitol) safely and effectively reduces the levels of intact parathyroid hormone in patients on hemodialysis," Journal of the American Society of Nephrology, vol. 9, no. 8, pp. 1427-1432, 1998.

[32] K. J. Martin, E. González, J. S. Lindberg et al., "Paricalcitol dosing according to body weight or severity of hyperparathyroidism: a double-blind, multicenter, randomized study," American Journal of Kidney Diseases, vol. 38, pp. S57-S63, 2001.

[33] S. M. Sprague, F. Llach, M. Amdahl, C. Taccetta, and D. Batlle, "Paricalcitol versus calcitriol in the treatment of secondary hyperparathyroidism," Kidney International, vol. 63, no. 4, pp. 1483-1490, 2003.

[34] D. G. Dobrez, A. Mathes, M. Amdahl, S. E. Marx, J. Z. Melnick, and S. M. Sprague, "Paricalcitol-treated patients experience improved hospitalization outcomes compared with calcitrioltreated patients in real-world clinical settings," Nephrology Dialysis Transplantation, vol. 19, no. 5, pp. 1174-1181, 2004.

[35] D. L. Andress, "Vitamin D in chronic kidney disease: a systemic role for selective vitamin D receptor activation," Kidney International, vol. 69, no. 1, pp. 33-43, 2006.

[36] A. J. Brown, J. Finch, and E. Slatopolsky, "Differential effects of 19-nor-1,25-dihydroxyvitamin $\mathrm{D}_{2}$ and 1,25-dihydroxyvitamin $\mathrm{D}_{3}$ on intestinal calcium and phosphate transport," Journal of
Laboratory and Clinical Medicine, vol. 139, no. 5, pp. 279-284, 2002.

[37] X. Li, W. Zheng, and Y. C. Li, "Altered gene expression profile in the kidney of vitamin D receptor knockout mice," Journal of Cellular Biochemistry, vol. 89, no. 4, pp. 709-719, 2003.

[38] S. Chen, J. Wu, J. C. Hsieh et al., "Suppression of ANP gene transcription by liganded vitamin D receptor: involvement of specific receptor domains," Hypertension, vol. 31, no. 6, pp. 1338-1342, 1998.

[39] T. Miyauchi, A. Fujimori, S. Maeda et al., "Chronic administration of an endothelin-a receptor antagonist improves exercise capacity in rats with myocardial infarctioninduced congestive heart failure," Journal of Cardiovascular Pharmacology, vol. 44, no. 1, pp. S64-S67, 2004.

[40] S. Lemmilä, H. Saha, V. Virtanen, I. Ala-Houhala, and A. Pasternack, "Effect of intravenous calcitriol on cardiac systolic and diastolic function in patients on hemodialysis," American Journal of Nephrology, vol. 18, no. 5, pp. 404-410, 1998.

[41] A. Gal-Moscovici and S. M. Sprague, "Use of vitamin D in chronic kidney disease patients," Kidney International, vol. 78, no. 2, pp. 146-151, 2010.

[42] C. W. Park, Y. S. Oh, Y. S. Shin et al., "Intravenous calcitriol regresses myocardial hypertrophy in hemodialysis patients with secondary hyperparathyroidism," American Journal of Kidney Diseases, vol. 33, no. 1, pp. 73-81, 1999.

[43] W. Xiang, J. Kong, S. Chen et al., "Cardiac hypertrophy in vitamin D receptor knockout mice: role of the systemic and cardiac renin-angiotensin systems," American Journal of Physiology, vol. 288, no. 1, pp. E125-E132, 2005.

[44] L. M. Resnick, F. B. Muller, and J. H. Laragh, "Calciumregulating hormones in essential hypertension: relation to plasma renin activity and sodium metabolism," Annals of Internal Medicine, vol. 105, no. 5, pp. 649-654, 1986. 


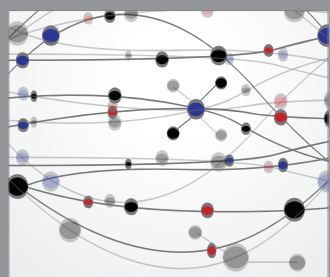

The Scientific World Journal
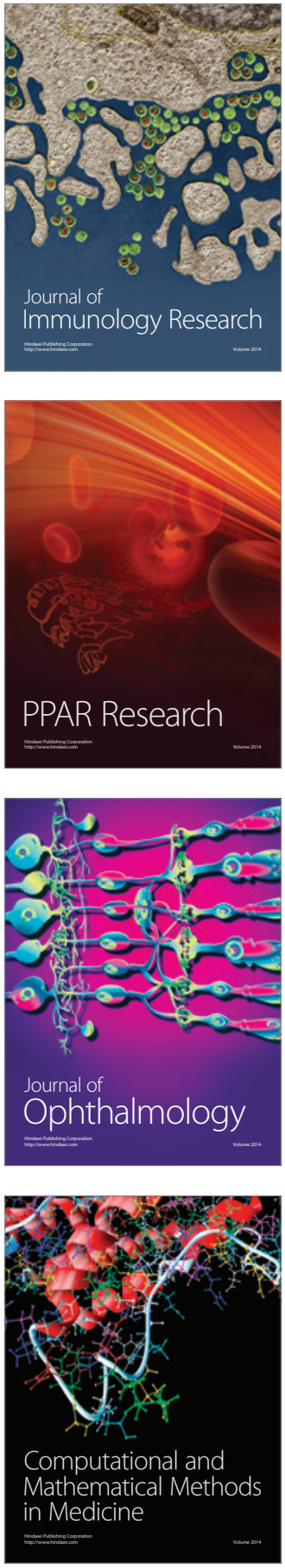

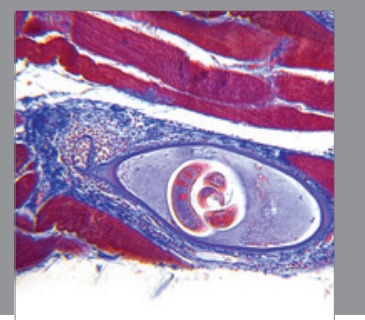

Gastroenterology

Research and Practice
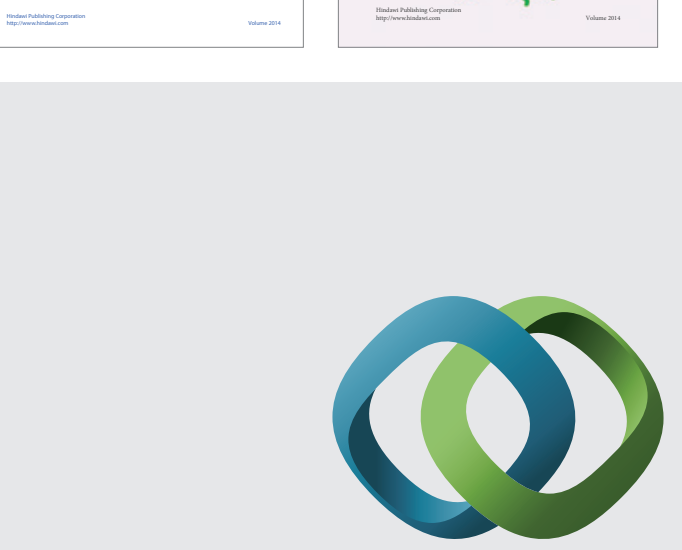

\section{Hindawi}

Submit your manuscripts at

http://www.hindawi.com
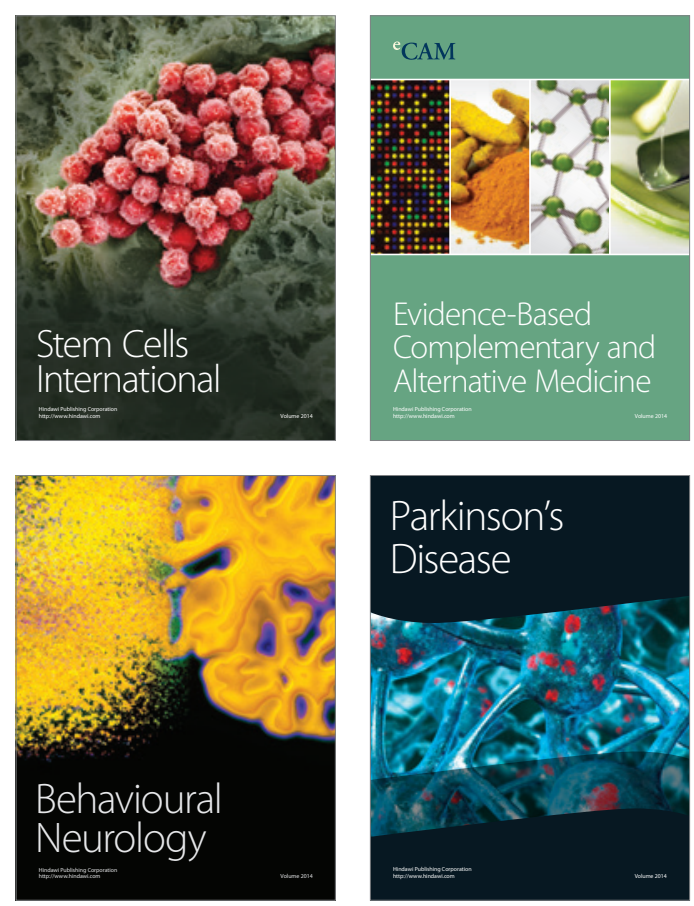

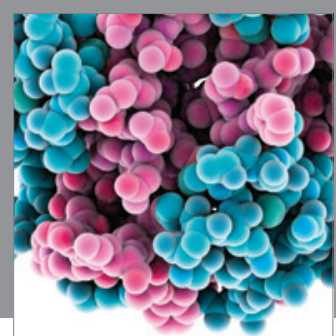

Journal of
Diabetes Research

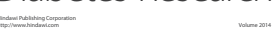

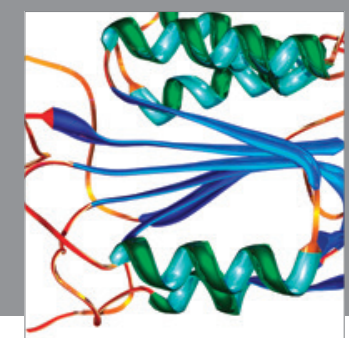

Disease Markers
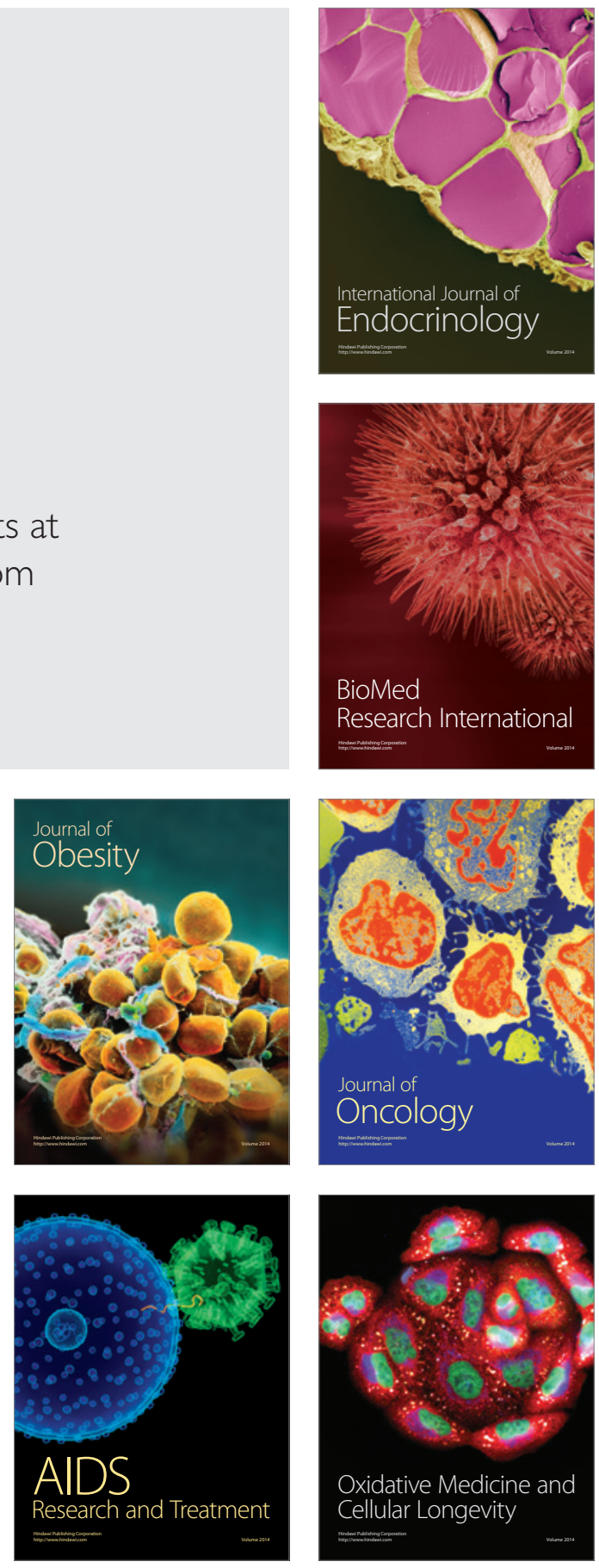